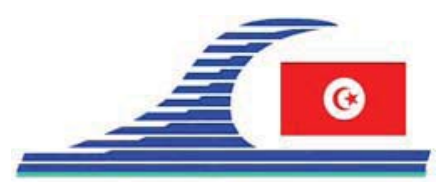

\title{
Interaction entre la houle et une plaque immergée en présence d'un courant
}

\author{
Vincent REY $^{1}$, Julien TOUBOUL ${ }^{1}$
}

1. Université du Sud - Toulon - Var, Laboratoire de Sondages Electromagnétiques de l'Environnement Terrestre, UMR 6017. BP 20132, 83957 La Garde Cedex, France. Julien.touboul@univ-tln.fr

\section{Résumé :}

Le but de ce travail expérimental était d'étudier, pour une houle d'incidence normale, l'influence d'un courant sur les interactions entre une houle régulière ou irrégulière et une plaque immergée. Les études ont été menées pour des courants homogènes de $0.3 \mathrm{~m} / \mathrm{s}$ dans le sens de propagation de la houle. Les résultats expérimentaux de réflexion en fonction de la période de la houle (dans un repère fixe) et pour différentes amplitudes ont montré une influence du courant de l'ordre de 15\% d'une part sur les positions des maxima et des minima de réflexion de la houle, et d'autre part sur leur intensité. Les positions des maxima d'énergie réfléchie sont donc peu affectées tandis que l'énergie réfléchie est moindre en présence d'un courant. L'intensité des efforts verticaux sur la plaque est inférieure d'environ $20 \%$ et plus homogène sur la gamme de période utilisée en présence d'un courant. En outre, les réflexions et efforts mesurés pour différentes conditions de houles (régulières, irrégulières) montrent un comportement des houles très correctement décrit par une approche linéaire.

\section{Mots-clés :}

Génie côtier - Réflexion - Efforts - Houle - Plaque plane - Courant

\section{Introduction}

En zone côtière, la houle peut être partiellement réfléchie par des variations rapides du fond. Ce «pouvoir de réflexion» de variations du fond a conduit à de nombreuses études et à la réalisation d'ouvrages côtiers immergés destinés à protéger des aménagements ou sites naturels de l'impact de la houle par réflexion partielle de son énergie (voir par exemple REY (1992), REY (1995)). Pour des zones côtières où la profondeur d'eau croit rapidement, des ouvrages de type plaque immergée ont été proposés pour la protection côtière. Les études de leur pouvoir de réflexion en fonction de la valeur relative de la longueur d'onde de l'onde par rapport à la longueur de la plaque à partir de modèles parfaits et linéaires ont montré la présence de maxima et minima de réflexion (PATARAPANICH,1984; STUROVA, 1991). Ce caractère oscillant de l'intensité de l'énergie réfléchie est du à un phénomène d'interférence au dessus de la plaque. Les aspects non-linéaires ont fait l'objet d'études plus récentes

$$
\text { DOI: } 10.5150 / \mathrm{cmcm} .2009 .014
$$


(BROSSARD \& CHAGDALI, 2001). En zone côtière, des courants de l'ordre de 0.1 à 1 $\mathrm{m} / \mathrm{s}$ sont souvent observés, induits par la marée ou par le déferlement des houles. Ils peuvent avoir un rôle important sur la vitesse de propagation et la direction de la houle (PHILLIPS, 1977), et par conséquent peuvent avoir une influence sur l'efficacité des brise-lames (REY et al., 2002). Le travail expérimental présenté ici a été réalisé dans le Bassin de Génie Océanique (BGO) FIRST, près de Toulon, France. Il consistait en l'étude de l'impact d'une plaque immergée sur la propagation de houles régulières ou irrégulières en présence d'un courant homogène. Les effets de la plaque sur la réflexion de la houle ainsi que les efforts subis par la plaque sont calculés et discutés.

\section{Résultats et interprétations}

\subsection{Coefficients de réflexion}

Les coefficients de réflexion ont été calculés par la méthode à trois sondes (REY et al. 2002) à partir des sondes amont. Nous avons représenté sur la Figure 1 les coefficients de réflexion en fonction de la période, en l'absence de courant (à gauche) et en présence d'un courant (à droite). La valeur théorique en l'absence de courant est présentée en trait plein. Les rectangles creux correspondent aux houles monochromatiques. Les spectres de réflexion (racine du rapport entre énergie réfléchie et énergie incidente par unité de fréquence) sont présentés pour les différentes périodes pic et valeurs de étudiées. Deux types de spectres de JONSWAP ont été générés, le premier de bande étroite $(\gamma=3.3)$, le second de bande plus large $(\gamma=1)$.
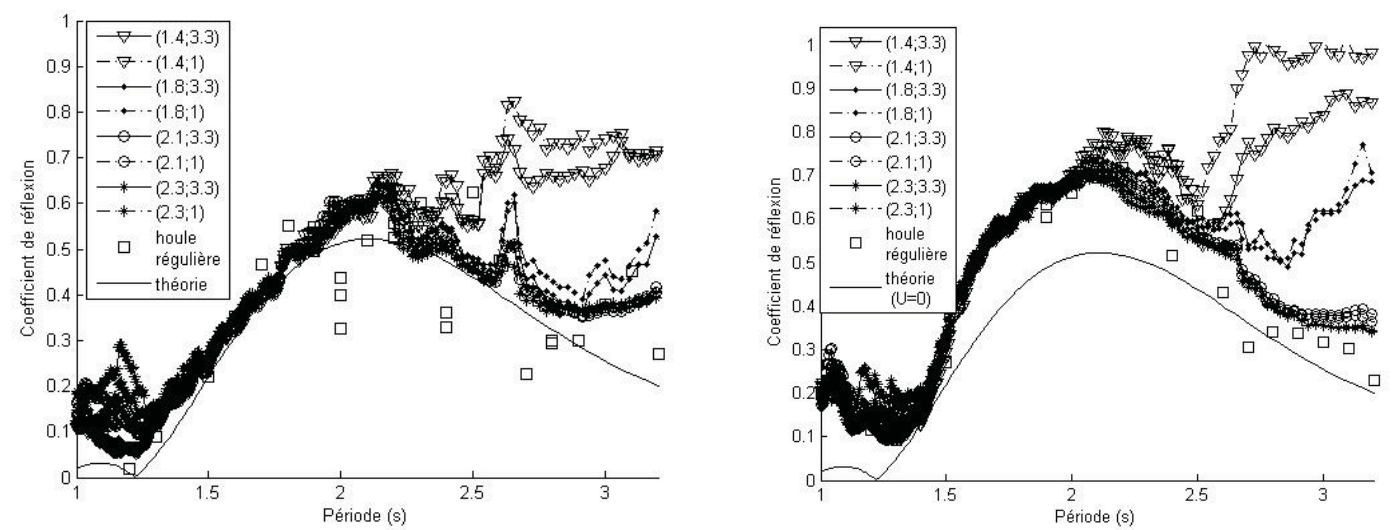

Figure 1. Coefficients de réflexion obtenus pour les houles régulières et irrégulières en l'absence de courant (à gauche), et en présence d'un courant $U=0.3 \mathrm{~m} / \mathrm{s}$ (à droite).

Le calcul théorique en l'absence de courant $(\mathrm{U}=0)$ est basé sur une formulation intégrale des conditions de continuité de vitesse et de pression aux abscisses $x=0$ et $x=L$. Il suppose un écoulement potentiel, et des ondes de faible amplitude. Il tient compte de la présence de modes évanescents au voisinage de la structure. La courbe théorique fait apparaitre des maxima et minima de réflexion, qui traduisent un phénomène d'interférence de l'onde au dessus de la plaque. 
On observe sur la Figure 1 (à gauche) un bon accord entre théorie et expériences pour les houles régulières et irrégulières. En particulier, pour les houles irrégulières, à l'exception des plus basses périodes pics ( $\mathrm{T}_{\mathrm{p}}=1.4$ et $\left.1.8 \mathrm{~s}\right)$, le spectre de réflexion est en très bon accord avec la théorie. Le très bon accord avec la théorie souligne la linéarité $\mathrm{du}$ processus sur une large gamme de fréquences et d'amplitudes. Le désaccord aux grandes périodes pour les houles de périodes pic $\mathrm{T}_{\mathrm{p}}=1.4$ et $1.8 \mathrm{~s}$ peut s'expliquer par la très faible énergie aux plus basses fréquences, et à une moins bonne absorption par la plage.

\subsection{Efforts verticaux sur la plaque}

Les efforts verticaux ont été calculés à partir des données des capteurs situés sur les parties supérieure et inférieure de la plaque. Les efforts sont présentés sur la Figure 2, respectivement en l'absence de courant (à gauche) et en présence d'un courant (à droite). Ils sont exprimés en daN par unité de largeur de la plaque (suivant la direction $0 \mathrm{y}$, normale à l'axe de propagation) par unité d'amplitude de la houle (ou de sa composante spectrale pour les houles irrégulières). On observe que l'intensité des efforts augmente régulièrement avec la période, avec un maximum de l'ordre de 1200 $\mathrm{daN} / \mathrm{m} 2$ pour des périodes comprises entre 2 et $2.7 \mathrm{~s}$. La surestimation observée du coefficient de réflexion aux grandes périodes pour les houles irrégulières de faible période pic (1.4 et $1.8 \mathrm{~s}$ ) n'a que peu d'impact sur l'intensité relative de la force verticale. L'intensité des forces de pression est plus homogène en présence d'un courant, puisque qu'elle reste de l'ordre de $1000 \mathrm{daN} / \mathrm{m} 2$ pour des périodes comprises entre 1.3 et $2.7 \mathrm{~s}$. Ici encore, on observe le caractère linéaire des efforts verticaux exercés sur la plaque par la houle.
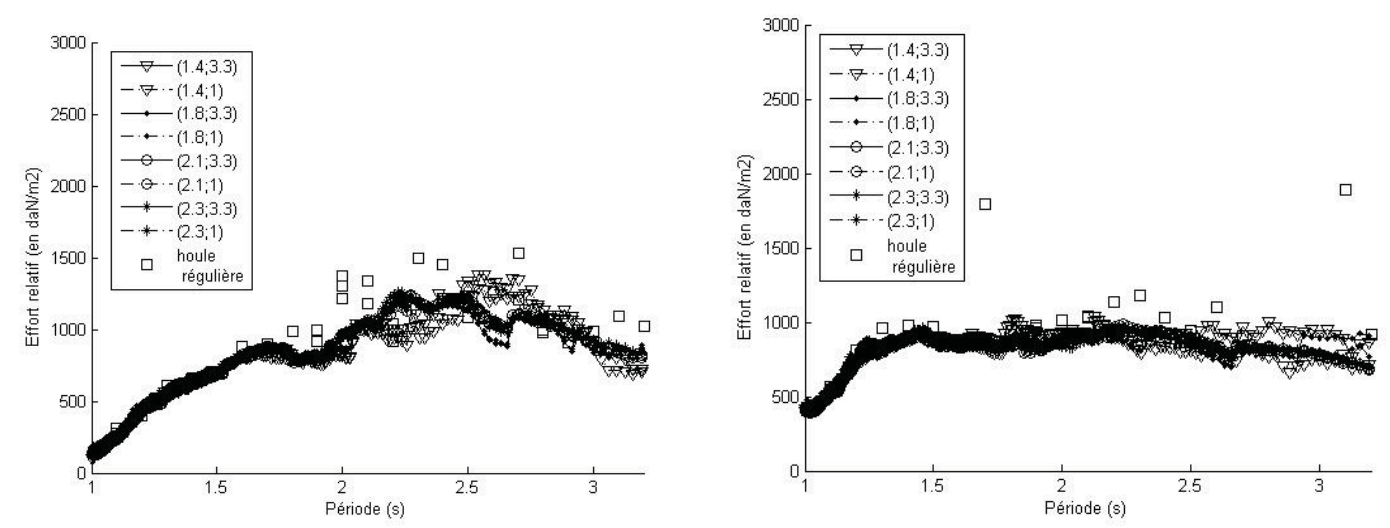

Figure 2. Efforts relatifs exercés sur la plaque par des houles régulières et irrégulières en l'absence de courant (à gauche), et en présence d'un courant $U=0.3 \mathrm{~m} / \mathrm{s}$ (à droite).

\section{Discussion et conclusion}

Les expériences présentées et analysées dans ce travail ont été menées dans un bassin d'essai qui présentait l'avantage de générer des houles superposées à des courants. Ce 
travail a permis de montrer qu'en présence d'un courant superposé à une houle incidente, l'amplitude réfléchie par une structure immergée de type plaque plane est supérieur à celle mesurée en l'absence de courant, à cause de l'effet doppler induit sur la houle réfléchie se propageant à contre courant. D'un point de vue énergétique, cette tendance s'inverse, l'énergie réfléchie en présence de courant étant plus faible qu'en l'absence de courant. La plage d'efficacité de la plaque reste inchangée. L'étude du processus d'interférence au dessus de la plaque, basé sur la théorie linéaire des ondes, supposées planes, nous donne une information quantitative des maxima et minima de réflexion, comme déjà observé par PATARAPANICH (1984) en l'absence de courant. Le poids des modes locaux sur l'évolution de la phase de l'onde au voisinage des discontinuités reste donc très inférieur à celui observé pour des obstacles rectangulaires immergés (REY et al., 1992), où l'étude basée sur l'approximation onde plane ne permettait qu'une estimation qualitative de la position des extrema. En outre, les résultats pour différentes amplitudes à fréquence donnée montrent une faible influence de l'amplitude sur le coefficient de réflexion, ce qui d'une part conforte les études théoriques basées sur des houles linéaires (ou composées de la superposition linéaire de houles linéaires) et d'autre part nous permet de conforter l'hypothèse linéaire sur laquelle est basée la méthode d'analyse des signaux de houle et de pression.

Enfin l'analyse présentée se limite aux calculs de réflexion et d'effort. Les analyses plus locales des signaux à partir des ondes disposées au voisinage de la structure permettront une étude plus complète du rôle des modes locaux sur l'hydrodynamique.

\section{Remerciements.}

Les auteurs remercient le Conseil Général du Var pour son aide financière qui a permis la réalisation des expériences au BGO First en 2009.

\section{Références bibliographiques}

BROSSARD J., CHAGDALI M. (2001). Experimental investigation of the harmonic generation by waves over a submerged plate. Coastal Engineering, (42), pp 277-290.

PATARAPANICH M. (1984). Maximum and zero reflection from submerged plate". Journal of Waterway, Port, Coastal and Ocean Engineering, (110, 2), pp 171-181.

PHILLIPS O.M. (1977). The Dynamics of the Upper Ocean. Cambridge University Press, 2nd edition. REY V. (1992). Propagation and local behavior of normally incident gravity waves over varying topography. European Journal of Mechanics B/Fluids, (11), pp 213-232.

REY V. (1995). A note on the scattering of obliquely incident surface gravity waves by cylindrical obstacles in waters of finite depth. European Journal of Mechanics B/Fluids, (14, 1), pp 207-216.

REY V., BELZONS M., GUAZZELLI E. (1992). Propagation of surface gravity waves over a rectangular submerged bar. J. Fluid Mech., (235), pp 453-479.

REY V., CAPOBIANCO R., DULOU C. (2002). Wave scattering by a submerged plate in presence of a steady uniform current. Coastal Engineering, (47), pp 27-34.

STUROVA V. (1991). Propagation of plane surface waves over an underwater obstacle and a submerged plate. Journal of applied mechanics and technical physics, (32, 3), pp 453-479. 